\title{
An Improved Image Compression Algorithm Based on Embedded Zerotree Wavelets Transform
}

\author{
Yanjun Liu and Zupeng Liu
}

\begin{abstract}
After analyzing the basic theory of embedded zerotree wavelet (EZW) coding algorithm, this paper brings forward an improved method which rounds down the wavelet coefficients and sets the coefficients appearing more frequent as zero, so as to reduce scan frequency in algorithm and save the storage space. Experimental results show that this algorithm improves the coding efficiency on the one hand; and on the other hand the quality of reconstructed image is also not significantly decreased.
\end{abstract}

Index Terms-Frequency, image compression, wavelet coefficients, embedded zerotree wavelets (EZW)

\section{INTRODUCTION}

In recent years, with the popularization of multimedia application and the development of digital video technology, as well as the increase of image browsing and transmission in internet, the demand for image processing technology is more and more high, to a picture, the image processing includes: image compression, image enhancement, and the restoration of image noise etc. It is very important as far as possible to reduce the image storage space in the process of image collection, storage and transform. Therefore, the improvement of current image compression technology has become the important problem in current information era to be resolved. Wavelet image compression coding with the advantages of its high compression rate and higher quality of image restoration, makes it have great application value and broad prospect in the image compression field.

\section{EZW CODING ALGORITHM}

In 1993 J.M. Shapiro proposed a kind of high-efficiency wavelet image compression algorithm, which was called embedded wavelet zerotree algorithm[1-4], the coding is being recognized as a very effective image coding algorithm in public, in current which is thought as one of the best method in the field of static image transformation compression coding. The bit in the bittorrent obtained from this algorithm is sorted according to the importance of coefficients. The coder can end coding at any time at any point with this algorithm, which will make coding accurately reach the expected goal of the bit rate, and at this moment it still can produce excellent image effect, the characteristics of

Manuscript received April 15, 2012; revised June 2, 2012.

Yanjun Liu is with the College of Computer and Information Engineering, Xinxiang University, Xinxiang, 453003, China (e-mail: lyjabb@163.com)

Zupeng Liu is with Department of Electronic and Communication Engineering, Henan Mechanical and Electrical Engineering College, Xinxiang, 453000, China. this algorithm does not require the drill, prior storage of formats streaming, and any earlier stage knowledge about image source.

\section{A. The Ideological Characteristics of Encoding Algorithm}

A piece of image does not compress the original image after wavelet transformation, but the energy of the whole image is redistributed, and carrying on the compression must also be quantitatively and entropy coding. In general, in the process of wavelet image compression quantification is one of the most crucial parts, it preferably organizes the wavelet coefficients of image to realize effective compression; entropy coding is forming wavelet coefficients coding after quantification to output bittorrent, the specific process is shown in figure 1 below.

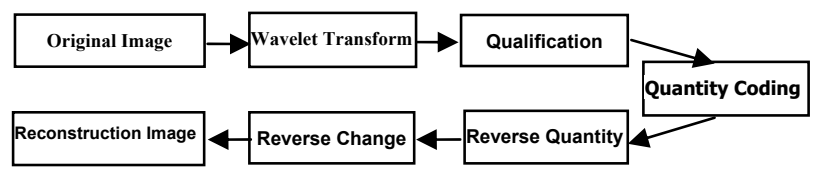

Fig. 1. The reconstruction process of original image.

In coding algorithm, there are two definitions needing to make clear:

1) The embedded code refers to the low bit encode output by encoder embedded in the start part of code streaming, that is, from the start point of embedded streaming to some position after this period of streaming is picked out, it equivalents to a code streaming with lower bit rate, it can decode and reconstruct the whole image, comparing with the original code streaming, the image decoded by this part of streaming has lower resolution and complete image effects.

2) Zerotree refers to under a given threshold, the coefficient value of root node and all its descendant nodes are invalid values, this root node is called zerotree root. The zerotree root refers the tree starting from the root node is zero tree, and at the same time the zerotree is not a subset of bigger zerotree, that is, the node is called zerotree root, its father node is not zerotree root.

In EZW algorithm, the realization of the embedded code flow is implemented by zerotree structure combing with successive approximation transmission. The algorithm uses the method of zerotree quantitative successive approximation, makes full use of correlation among the sub-bands of image wavelet coefficients, low bit rate has high signal-to-noise ratio. The basic idea of zerotree quantification algorithm is using the zerotree data structure while quantifying wavelet coefficients, to make full use of the time-frequency locality of wavelet transform and the correlation among sub-band of mining transform coefficients. The coding of this algorithm is according to $Z$ type to do successive scan, in a given 
threshold value $\mathrm{T}$, each wavelet coefficient is divided into the positive important coefficient POS (P), the negative important coefficient NEG $(\mathrm{N})$, isolated zero $\mathrm{IZ}(\mathrm{Z})$ and zerotree root ZTR $(T)$. In a given threshold value the algorithm experiences two processes: the main scanning and vice scanning, and then the threshold value drops to $1 / 2$, in the condition of lower threshold value, which realizes the successive approximation embedded coding.

\section{B. The Process of Coding Algorithm}

1) Determine the initial threshold, $T_{0}$ is used to presents initial threshold: $T_{0}=2^{\text {floor }\{\log 2(\max (|r(i, j)|))\}}$, in the expression, floor \{\} represents that rounded down to the integer, $r(i, j)$ represents the wavelet coefficient value, and later for each scan, the threshold is cut by half.

2) Form main table according " $Z$ " scanning sequence, again according to amplitude encoding process form vice table, the scan process is shown in Fig. 2.

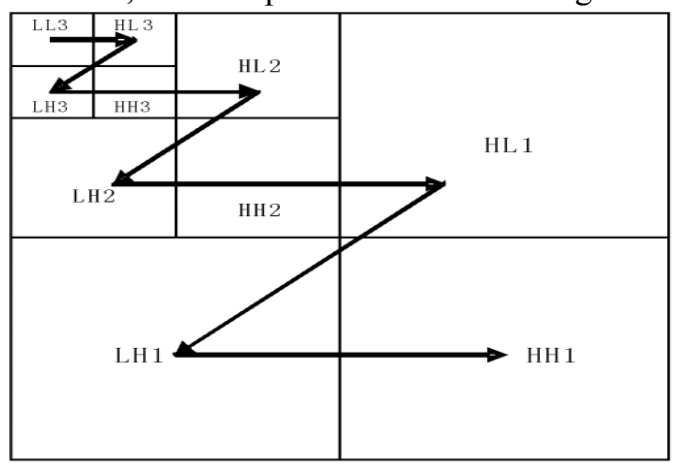

Fig. 2. "Z" type image scanning.

The main scan is used to complete the following tasks:

According to the output code flows after scanning wavelet coefficients, suppose coefficient is $\mathrm{X}[\mathrm{i}]$, the threshold is $\mathrm{T}$, it will follow the following rules:

If $X[i]>T$, the output is $\mathrm{P}$;

If $X[i]<-T$, the output is N;

If $X[i] \mid<T$, and there is an important coefficient in the offspring coefficient, then output $\mathrm{Z}$;

If $|X[i]|<T$, and there is no important coefficient in the offspring coefficient, then output $\mathrm{T}$;

The positive and negative important coefficient will be extracted, take its absolute value and put in the vice table.

The position of important coefficient in the main table is set to zero.

Auxiliary scanning is used to complete the following tasks:

For the important coefficient after main scan to do refining coding, according to the coefficient in vice table to output 0 or 1 , specifically follow the rules:

When the absolute value of important coefficient is located between $\mathrm{T} \sim 3 / 2 \mathrm{~T}$, it is represented as 0 or 1 ;

When the absolute value of important coefficient is located between $3 / 2 \mathrm{~T} \sim 2 \mathrm{~T}$, it is represented as 1 or 0 ;

When the threshold value reduces to 1 , according to the code stream output by main scan and vice scan all the coefficient value can be presented. code stream output by main scan and vice scan all the coefficient value can be presented.

Finally the output code flow needs entropy coding, the arithmetic coding is generally adopted. Because each round of threshold is different, finally in the vice table, the non-zero point listed in the preceding is more relative important than behind. In this judgment there exist only four kinds of symbols: positive value, negative value, zero root, and isolated zero, so that it can realize the compression of bits. These four kinds of symbols and the symbol in nonzero numerical range use entropy coding in accordance with the size of probability to realize compression, to achieve the requirement of low bit rate. Since in each sub-band the meaningful coefficient is according to the principle from father system to child system and the priority of important value arranged in vice table, so according to the compression requirement to obtain important value automatically.

3) According to the importance principle to adjust the order of amplitude in the vice table.

4) The values in main table and vice table are sent to entropy encoder to do adaptive arithmetic coding output.

5) While reaching target bit rate the coding stops, otherwise, repeat steps 2), 3).

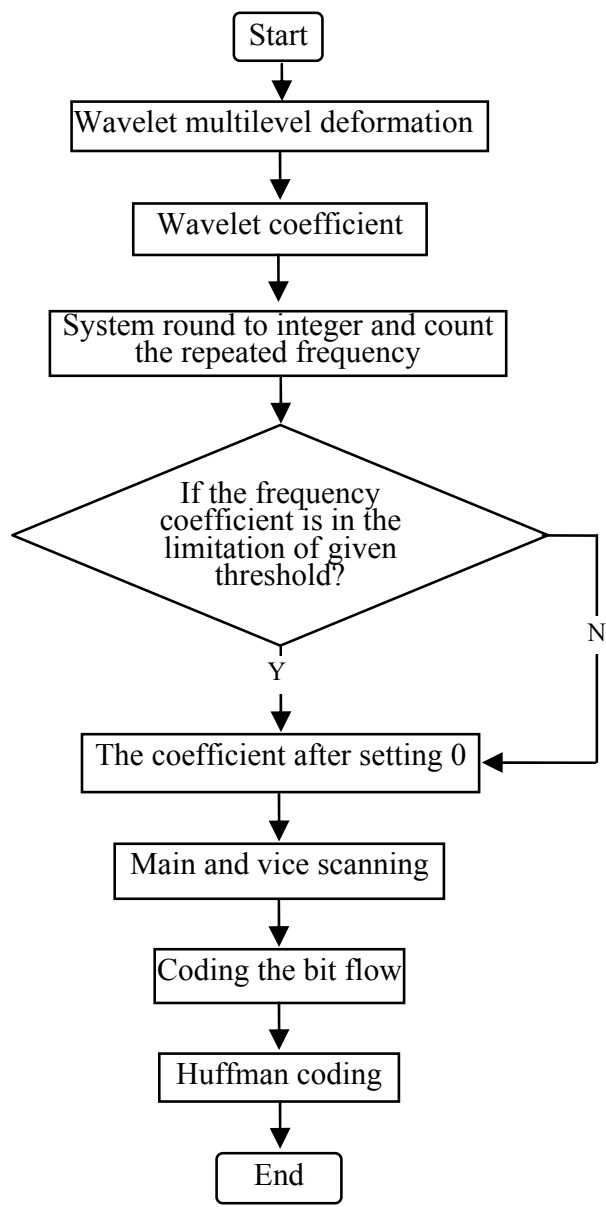

Fig. 3. The improved algorithm code flow chart.

\section{IMPROVED CODING AlgORITHM}

When the original algorithm do quantification in the tree 
structure, the value of threshold is according to the negative power level to reduce gradually, and every reduce once, the high frequency sub-image is need to do zerotree classification, the main table and vice table are respectively do multi zero searching and judgment processing, which will consume a lot of time, and compression effect is limited. After image signal does multistage wavelet transform, the most of the energy is focused in the low frequency part, and in low frequency level wavelet coefficients shows larger amplitude, which is far bigger than other wavelet coefficients with high frequency sub-band, reflects the main characteristics of image signal, and plays a key role to the restore of image quality.

The improved method proposed in this paper is based on wavelet transform coefficients for related statistical analysis, there exist a number of repeated coefficients and most of them are focused in the high frequency part after wavelet coefficients round down the integer. In the premise of keeping the quality of reconstructed image almost same, these non-important coefficients unifying into zero can reduce the scanning number in the given adjustment frequency range within high and low threshold, so as to improve the coding efficiency. The quantified coefficients adopts Huffman encoding in order to achieve the purpose of efficient compression to do prejudice compression, the flow chart is illustrated in figure 3 below.

The process is as follows:

1) Suppose after the multi-level wavelet transform, the wavelet coefficient is $\mathrm{C}(\mathrm{i}, \mathrm{j})$, its round down integer is $R(i, j)=|C(i, j)|$;

2) Statistics the frequency of repeat coefficient $\mathrm{N}(\mathrm{N}$ is the number of duplicate values appearing in coefficients) in the coefficient matrix after taking integer;

3) Set the threshold range $[\mathrm{M}, \mathrm{N}]$ of the zero coefficient (based on image quality to do adjustment, in the experimental simulation it is [-24,24]), and determine if the repeated frequency coefficient is in the range of given threshold, if it is then set to zero, and vice versa does not change;

4) Execute main and sub scanning, quantization and Huffman coding to formative changed wavelet coefficient matrix.

\section{The Simulation Results}

If you are using Word, use either the Microsoft Equation Editor or the MathType add-on (http://www.mathtype.com) for equations in your paper (Insert | Object | Create New | Microsoft Equation or MathType Equation). "Float over text" should not be selected.

This simulation EZW coding algorithm gives an example of bitmap (Figure 1, bmp format, wavelet basis is bior4.4, wavelet transform is eight level) with resolution lena $(256 \times 256)$ on the desktop which main clock is $2000 \mathrm{MHz}$ to achieve the improved algorithm's simulation using Matlab. Here are the image comparisons of the experimental reconstruction between the original EZW algorithm and improved EZW algorithm, Figure 5 and Figure 6 present the comparison of experimental performance indexes shown in Table I.

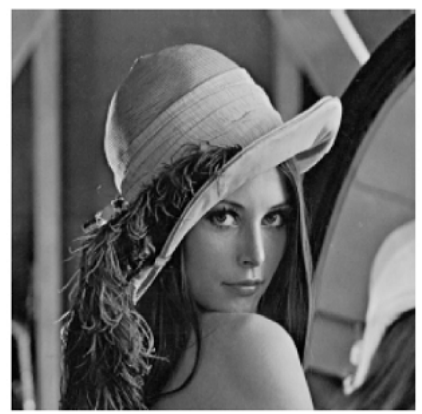

Fig. 4. Original image lena $(256 \times 256)$.

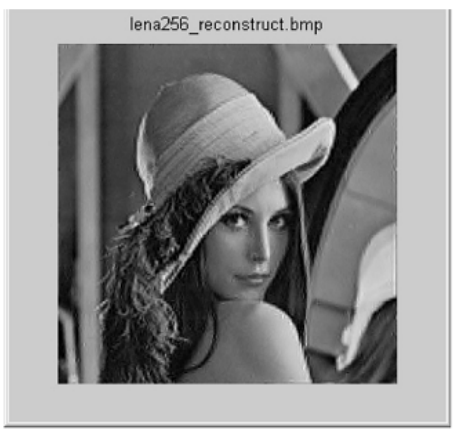

Fig. 5. Lena reconstructed image of EZW algorithm.

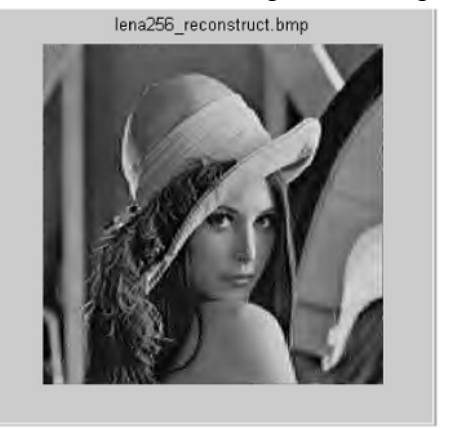

Fig. 6. Lena reconstructed image of improved EZW algorithm.

TABLE I: THE PERFormance COMPARISONS BETWEEN ORIGINAL AND IMPROVED EZW ALGORITHM

\begin{tabular}{cccc}
\hline \hline $\begin{array}{c}\text { Name of } \\
\text { algorithm }\end{array}$ & $\begin{array}{c}\text { Coding rate } \\
\text { /bpp }\end{array}$ & $\begin{array}{c}\text { Total bit } \\
\text { /bit }\end{array}$ & $\begin{array}{c}\text { Peak value } \\
\text { signal-to-noise ratio } \\
/ \mathrm{dB}\end{array}$ \\
\hline EZW algorithm & 1.07125 & 548 & 27.76 \\
$\begin{array}{c}\text { Improved EZW } \\
\text { algorithm }\end{array}$ & 0.12875 & 66 & 27.75 \\
\hline \hline
\end{tabular}

Through the above comparison, the improved EZW algorithm has better compression effect than original EZW algorithm. The image coding bits is almost $1 / 8$ of the original EZW algorithm; meanwhile the image reconstruction quality changes little.

\section{CONCLUSIONS}

This paper presents an improved embedded zerotree coding algorithm (IEZW) about compression effect of images. This algorithm through analysis of coefficients characteristics after the wavelet transform, deals with the duplicate data in wavelet coefficients, makes the number of scans reduced, and thus shortens the output code streaming, according to the encoding and the requirements to restore the image quality to end coding at any time, which has good 
compression effect. The experiment proves this improved algorithm is feasible in the aspect of increasing compression efficiency, and the quality of image reconstruction has no significant decline.

\section{REFERENCES}

[1] J. Chen and J. Yang, "Improved Embedded Zerotree Coding Algorithm," Computer Engineering, vol. 36, no. 2, pp. 209-213, 2010.

[2] L. Zhao and H. Xue, "Research of Image Registration Method Based on Wavelet Decomposition and the Maximum Mutual Information," Shanxi Institute of Technology: Natural Science, vol. 26, no.3, pp. 22 $-26,2010$.

[3] F. Zhang and G. Zhu, "Based Pan-Fibonacci Web Images Fast Rendering," Modern Electronic Technology, 2009, vol. 32, no. 16, pp.103-106.

[4] L. Li, "Medical Image Compression Techniques Based on Wavelet Transform," Computer and Digital Engineering, vol. 37, no. 2, pp. 136-138, 2009.

[5] T, Cheng, "Embedded Image Coding Using Zerotree of Wavelet Coefficient," IEEE Trans on Signal Processing, vol. 41, no. 12, pp. 3445-3462, 1993.

[6] Y. Sun, "Wavelet analysis and its application," Beijing: Mechanical Industry Press, 2005.

[7] Gonzalez, Digital Image Processing. Qiuqi Ruan, translation. The 2nd version. Beijing: Electronic Industry Press, 2005.

[8] B. Xue and X. Gu, "Embedded Zerotree Wavelet Coding Algorithm and Applied Research," Chengdu: University of Electronic Science and Technology, 2008.

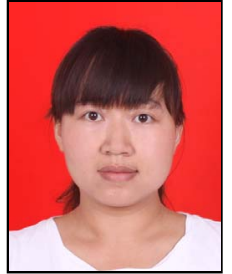

Yan Jun Liu was born in China on September 20, 1977. She incepted bachelor's degree of the application of electronic technology from The Information Engineering University of the PLA in Henan, China, 2001. And she incepted master's degree of software engineering from Huazhong University of Science and Technology in Hubei, China, 2007.

She is working as a LECTURER in the Xinxiang University in Henan, China now, and she started this job since 2001 after she earned her bachelor degree. She involved in writing several textbooks: New Edition of the Visual Basic Program Design Course (Beijing, China. Aviation Industry Press, 2005), and New Edition of the Fundamental Course for the Computer Science (Beijing, China. Aviation Industry Press, 2005). Her current research area focuses on the embedded system study. Ms. Liu: a member of IACSIT.

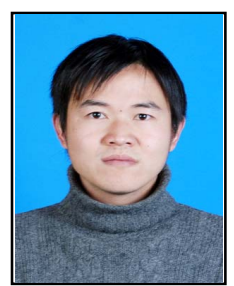

Zupeng Liu was born in China on February 2, 1980. $\mathrm{He}$ incepted bachelor's degree of the Electronic Information Engineering from Henan Normal University in Henan, China, 2003. And He incepted master's degree of Control Engineering from Wuhan University of Technology in Hubei, China, 2008.

$\mathrm{He}$ is working as a LECTURER in Henan Mechanical and Electrical Engineering College in Henan, China now and he started this job since 2004 after he earned his bachelor degree. His current research area focuses on the embedded system study. Mr. Liu: a member of IACSIT. 